Pour une histoire renouvelée des élèves (XVle$\mathrm{XXe}$ siècles). Volume 2 : sources et méthodes

\title{
Écrire l'histoire des élèves : problèmes de sources et de méthodes
}

A renewed history of students: sources and methods

Jean-François Condette et Véronique Castagnet-Lars

\section{OpenEdition}

Journals

Édition électronique

URL : https://journals.openedition.org/histoire-education/4334

DOI : $10.4000 /$ histoire-education.4334

ISSN : 2102-5452

Éditeur

ENS Éditions

Édition imprimée

Date de publication : 15 janvier 2019

Pagination : $9-25$

ISBN : 979-10-362-0144-8

ISSN : 0221-6280

Référence électronique

Jean-François Condette et Véronique Castagnet-Lars, «Écrire l'histoire des élèves : problèmes de sources et de méthodes », Histoire de l'éducation [En ligne], 151 | 2019, mis en ligne le 01 janvier 2021, consulté le 06 janvier 2022. URL : http://journals.openedition.org/histoire-education/4334 ; DOI : https://doi.org/10.4000/histoire-education.4334 


\title{
Écrire l'histoire des élèves : problèmes de sources et de méthodes
}

\author{
Jean-François Condette et Véronique Castagnet-Lars
}

Dès 1974, dans un article de la Revue française de pédagogie sur "Les sources de l'histoire de l'éducation et leur exploitation "1, Dominique Julia dressait un intéressant inventaire des sources disponibles et des pistes de recherches offertes pour la période moderne et le XIX ${ }^{\mathrm{e}}$ siècle. Présentant d'abord les enquêtes statistiques sur l'école primaire et l'enseignement secondaire, en un temps où l'histoire sérielle dominait encore le paysage historiographique, puis les sources permettant de "poser la question du recrutement aussi bien des élèves que des maîtres", il terminait sa contribution par les moyens dont dispose l'historien pour entreprendre l'étude de la vie scolaire. Insistant sur l'importance des travaux menés par l'Institut national d'études démographiques (INED), en particulier par le suivi de cohortes d'élèves, regroupés dans le recueil "Population" et l'enseignement ${ }^{2}$ (1960), il montrait alors que cette question du parcours des élèves et l'étude de leurs caractéristiques sociales sont délicates à mener pour les périodes antérieures, les listes et registres d'élèves étant particulièrement rares $^{3}$. La tenue de ces registres "laisse aussi beaucoup à désirer" alors que l'on se trouve également confronté à de délicates questions méthodologiques dans la conception de "la stratification socio-professionnelle des parents" sur la longue durée. Terminant par la vie scolaire, il insistait alors sur «la vision de l'enseignement par ses usagers ${ }^{4}$ " et, après avoir signalé la richesse des sources émanant des administrateurs et des enseignants, il notait à propos des élèves :

"Les témoignages qui nous ont été laissés sont le plus souvent indirects : et nous connaissons mieux les projections des adultes sur les enfants que l'expression des enfants eux-mêmes. Pourtant les correspondances des enfants

1 Dominique Julia, "Les sources de l'histoire de l'éducation et leur exploitation ", Revue française de pédagogie, n²7, 1974, p.22-42.

2 INED, "Population" et l'enseignement, Paris, Presses universitaires de France, 1970.

3 Dominique Julia, "Les sources de l'histoire de l'éducation...", art. cit., p. 26.

$4 \quad$ Ibid., p. 31. 
avec leurs parents ou avec leurs professeurs sont, malgré leur rareté, un lieu privilégié d'informations, parce que internes au champ pédagogique lui-même. Les mémoires constituent une autre source de renseignements et un corpus des passages concernant l'éducation des auteurs aurait sans nul doute un grand intérêt. Il ne faut pas oublier cependant qu'il s'agit là de la mémorisation tardive d'adultes vieillissants se penchant sur leur enfance et le travail du souvenir qui s'y opère idéalise quelque peu le tableau présenté. La même observation vaut pour les publications des associations amicales d'anciens élèves d'établissements qui ont fleuri au $\mathrm{XIX}^{\mathrm{e}}$ siècle et dans la première moitié $\mathrm{du} \mathrm{XX}^{\mathrm{e}}$ siècle et dont les annuaires relatent avec complaisance les festivités " 5 .

En 1992, dix-huit ans plus tard, Rebecca Rogers note dans l'avant-propos de son livre sur Les demoiselles de la Légion d'honneur :

"Comment retrouver l'individualité de mes sujets historiques? Frustration suprême d'en savoir autant sur l'héritage social et les itinéraires scolaires et si peu sur l'intériorisation de cette éducation et sur l'avenir des jeunes filles. C'est alors que je lus le journal d'Eugénie Servant, élève à la maison d'Écouen entre 1875 et 1881 . Il était rempli des menus faits de la vie au pensionnat et elle décrivait à merveille le quotidien de son existence. Document opaque en raison des nombreux interdits qu'elle intériorisait, cette pièce essentielle me donnait malgré tout la possibilité de restituer un peu de chair aux individus numérotés du fichier informatique ${ }^{6}$.

Dans le bilan historiographique proposé sur "l'éducation des filles : un siècle et demi d'historiographie" (2007), la même auteure relève, trente-trois ans après l'article de Dominique Julia, la rareté des travaux sur les élèves étudiés sous le prisme du genre. "La rareté des sources "classiques", des dossiers d'élèves en particulier, est notamment responsable de cette pénurie qui caractérise l'histoire de l'enseignement en général » ${ }^{7}$. En 2017 Antoine Prost, dans un article intitulé "Morphologie et sociologie des lycées et collèges (1930-1938)", opère le même constat, tout en livrant une étude très riche sur les élèves :

"Nous connaissons assez mal les élèves qui fréquentaient l'enseignement secondaire entre les deux guerres. Nous ne savons rien sur les collèges privés, qui accueillent pourtant à peine moins d'élèves que les établissements publics, et pour lesquels, si des sources existent peut-être encore, leur recherche s'ap-

$5 \quad$ Ibid., p. 31.

6 Rebecca Rogers, Les demoiselles de la Légion d'honneur, Paris, rééd. Perrin, 2006, "Avant-propos", p. 9 .

7 Rebecca Rogers, "L'éducation des filles : un siècle et demi d'historiographie" in Pierre Caspard, JeanNoël Luc, Rebecca Rogers (dir.), L'éducation des filles (XVIII ${ }^{\mathrm{e}} \mathrm{XXI}{ }^{\mathrm{e}}$ siècles). Hommage à Françoise Mayeur, Histoire de l'éducation, nº 115-116, 2007, p.37-79, (p.68). 
parenterait à celle d'une aiguille dans une meule de foin. Pour les lycées et collèges publics déjà, les archives susceptibles de nous renseigner sont rares " ${ }^{8}$.

Les difficultés archivistiques sont ici systématiquement mises en avant dans la volonté d'écrire une histoire renouvelée des élèves à la fois, en raison de la rareté et de la dimension lacunaire des sources et par leur spécificité, souvent relevée, puisqu'elles sont des traces indirectes, composées par des adultes pour d'autres adultes. Dans le même temps, les auteurs évoqués nous donnent cependant des pistes pour retrouver ces mêmes élèves, par le recours aux statistiques, par l'usage des écrits du for privé (lettres, journaux intimes, etc.) mais aussi par l'analyse des mémoires et souvenirs.

Les sources qui rendent possible une histoire renouvelée des élèves ainsi que leur traitement scientifique sont au cœur du présent numéro. Les cinq articles qui le composent couvrent ensemble une période étendue de l'Ancien Régime au second $\mathrm{XX}^{\mathrm{e}}$ siècle. On y voit l'historien chercher la trace des élèves et de leurs expériences au travers de sources diversifiées, puis travailler, selon ses approches spécifiques, sur ces matériaux rassemblés. Sources archivistiques, sources imprimées et enquêtes statistiques sont ici privilégiées, mais il existe bien évidemment d'autres approches tout aussi légitimes, fondées sur l'enquête orale ou l'usage des représentations iconographiques et filmiques. Ce numéro propose ainsi des études de cas précises et doit donc s'envisager comme la suite et l'illustration de la précédente livraison de la revue ( $\mathrm{n}^{\circ} 150$ ) consacrée à un bilan historiographique international.

\section{Des sources pour l'histoire des élèves}

L'histoire des élèves, qui s'est fortement enrichie ces dernières années, rencontre systématiquement un certain nombre de difficultés dans sa quête documentaire qui sont liées à la nature des sources mobilisées et de l'objet étudié.

\section{L'élève à l'arrière-plan des sources traditionnelles?}

Nous ne revenons pas sur la diversité des sources qui sont à la disposition de l'historien pour lui permettre d'évaluer la place et le rôle des élèves eux-mêmes dans le processus d’instruction, ces éléments ayant déjà été développés dans le

8 Antoine Prost, "Morphologie et sociologie des lycées et collèges (1930-1938) ", Histoire de l'éducation, $\mathrm{n}^{0} 146,2016$, p. 53-110. 
numéro précédent.L'examen des parcours scolaires, des choix d'établissements par la famille, au sein d'un territoire donné, l'étude des origines sociales des élèves selon les filières, les différences de parcours entre garçons et filles sont autant de thématiques fortes qui émergent des travaux actuels. Étudier l'élève en histoire de l'éducation n'est plus une brève parenthèse visant à donner un peu de chair à un récit, exemplifiant par une étude de cas, une démonstration plus générale sur l'École. L'élève n'est plus, non plus, une simple unité de compte dans des séries statistiques destinées à vérifier la bonne santé ou la crise des institutions scolaires. Il est bien devenu un objet d'études en soi. À chaque fois cependant, historiennes et historiens prennent le temps de mettre en garde le lecteur en signalant que les sources sont lacunaires, que l'objet d'étude qu'est "l'élève" se dérobe dans les sources, qu'il est évoqué partout mais présent spécifiquement nulle part. La synthèse due à François Grèzes-Rueff et à Jean Leduc, Histoire des élèves en France de l'Ancien Régime à nos jours (2007) ne propose ainsi pas de rubrique "sources" à côté de son imposante bibliographie et filmographie, travaillant surtout à partir des textes officiels et des représentations transmises par la littérature, la presse ou le cinéma ${ }^{9}$. De précieux instruments de recherche existent pourtant en histoire de l'éducation qui présentent un état précis des sources ${ }^{10}$.

Produites principalement par l'institution scolaire, c'est-à-dire par l'administration et ses agents, par les établissements et leurs responsables, les sources archivistiques donnent rarement à voir les élèves directement. Dans le rapport mensuel ou annuel du proviseur sur son lycée, le chef d'établissement tient à montrer à ses supérieurs hiérarchiques la bonne santé de son institution scolaire et sa bonne gestion. Il joint alors un tableau de statistiques sur la répartition des élèves, un autre sur les résultats aux examens tout en insistant sur le fait que la "discipline est bonne" et que les fortes têtes ont été sévèrement punies. Nous avons bien ici des "traces" sur les élèves, des indices, mais qui se doivent d'être complétés, croisés avec d'autres sources.

9 François Grezes-Rueff, Jean Leduc, Histoire des élèves en France de l'Ancien Régime à nos jours, Paris, Armand Colin, 2007.

10 Thérèse Charmasson (éd.), Archives et sources pour l'histoire de l'enseignement, Paris, Comité des travaux historiques et scientifique, 2005; Thérèse Charmasson (dir.), Histoire de l'enseignement, XIX ${ }^{e}-X X^{e}$ siècles. Guide du chercheur, Paris, Institut national de recherche pédagogique/Comité des travaux historiques et scientifiques, 2006 ; Jean-Noël Luc, Stéphanie Méchine, Emmanuelle Picard (dir.), Les archives universitaires. De nouvelles sources pour l'histoire de l'enseignement supérieur et de la recherche, Centre d'histoire du XIX siècle de l'université Paris Sorbonne, 2014 (disponible en ligne : https://halshs.archives-ouvertes.fr/halshs-01093146/document). 
Dans ce numéro, les textes de Jean Le Bihan et de Dominique Julia témoignent de la richesse des sources manuscrites existantes. Pour ce dernier, il s'agit de repérer les traces de l'activité pédagogique consignée par Madame de Genlis dans ses différents carnets, journaux... au prix d'une pérégrination entre la France et la Belgique, montrant ainsi que les archives des bibliothèques et les archives des musées offrent aussi de précieuses sources sur la connaissance des élèves de l'Ancien Régime. Après avoir étudié les lauréats des bourses d'État ${ }^{11}$, Jean Le Bihan s'intéresse ici aux "boursiers communaux de l'enseignement secondaire au XIX ${ }^{\mathrm{e}}$ siècle : un angle mort de l'historiographie des élèves". Il montre leur importance pour les lycées et collèges dans la mise en œuvre concrète du discours méritocratique peu à peu affirmé puis célébré sous la Troisième République. Les fonds des Archives nationales contiennent de nombreuses informations, tout comme les grandes enquêtes statistiques nationales, qui doivent cependant être complétées par les sources conservées dans les archives communales (délibérations du conseil municipal, etc.) et dans les archives départementales, parfois même les archives d'établissements (registres d'inscription, etc.). Il estime ainsi qu'un tiers des boursiers des collèges et des lycées de garçons en 1895 sont des boursiers communaux. Il est alors important de pouvoir saisir non seulement le résultat final (le nombre de bourses attribuées), qui peut être scruté depuis les documents de synthèse parisiens, mais aussi de comprendre les modalités de sélection des candidats, les finalités de ces bourses pour le financeur municipal et la surveillance qui s'opère sur ces élèves spécifiques.

Solenn Huitric, dans son étude sur "l'élève au prisme de l'administration : les collégiens et lycéens français entre 1830 et 1880" relève les difficultés à cerner les élèves dans les archives administratives. La priorité des documents rédigés par l'administration est d'informer les différents acteurs et relais de la chaîne hiérarchique patiemment construite au XIX ${ }^{\mathrm{e}}$ siècle de la situation de l'établissement à tel moment.Les recteurs ou le Ministère peuvent aussi décider, au-delà du rapport reçu, de se rendre sur place ou de dépêcher les corps d'inspections, ces actions déclenchant à leur tour l'écriture de rapports conservés dans les archives ${ }^{12}$. Les dépouillements croisés de sources, entre

11 Jean Le Bihan, "Le recrutement des boursiers des collèges royaux sous la monarchie de Juillet: nouveau regard à partir des ordonnances de nomination", Histoire de l'éducation, nº 144, 2015, p. 55-78.

12 Jean-François Condette, "Les personnels d'inspection en France au XIX siècle (1802-1914). Entre 
archives d'établissement, archives communales, départementales et nationales permettent alors de disposer d'informations complémentaires sur les élèves.

\section{Les écrits du for privé et les souvenirs d'élèves}

À côté des archives administratives, obéissant à des cadres prédéterminés, les récits de vie apparaissent comme des documents beaucoup moins normés et beaucoup plus spontanés qui reposent sur la volonté personnelle d'écrire ses souvenirs, englobant dès lors un récit sur les années d'enfance et de jeunesse et donc sur les années d'apprentissage, que celles-ci s'opèrent au sein de la famille, auprès de parents proches ou de précepteurs, ou qu'elles se réalisent dans une institution scolaire. Les écrits du for privé (lettres, carnet de bord, journal intime, etc. ${ }^{13}$ et les archives familiales ${ }^{14}$ sont alors des sources très riches pour enfin pouvoir percevoir la vie des élèves et leurs représentations. Ainsi l'exploitation des correspondances entre les élèves et leurs parents ou entre les parents et les chefs d'établissement ou les précepteurs sont riches en informations sur le soin apporté par les familles dans le choix du précepteur ou de l'établissement scolaire. La publication récente de Philippe Marchand, Donnez-moi des nouvelles... Collèges et collégiens à travers les correspondances familiales (1767-1787), dont on lira dans ce numéro une recension, démontre cette attention constante des parents envers leurs enfants placés comme élèves auprès d'un précepteur ou devenus pensionnaires au sein d'une institution scolaire ${ }^{15}$.

Les souvenirs et autobiographies abordant les années d'apprentissages, dans une partie de leur récit, forment un autre gisement documentaire important. C'est ce qu'a entrepris d'étudier depuis plusieurs années Pierre Caspard qui nous

affirmation des prérogatives de l'État et prise en compte des réalités scolaires locales" - "The inspection Staff in France in the Nineteenth Century (1802-1914): between Affirmation of the State's Prerogatives and local scholastic Context", Rivista di Storia dell'Educazione, numéro spécial dirigé par Juri Meda, Carla-Francesca Ghizzoni et Alberto Barausse, "Il campanile scolastico »: ripensando la dimensione locale nella ricerca storico-educativa - Repenser la dimension locale dans la recherche historique en éducation, 2018, p.79-97.

13 Jean-Pierre Bardet, Élisabeth Arnoul, François-Joseph Ruggiu (dir.), Les écrits du for privé en Europe du Moyen Âge à l'époque contemporaine. Enquêtes, analyses, publications, Pessac, Presses universitaires de Bordeaux, 2010; Jean-Paul Bardet, François-Joseph Ruggiu (dir.), Au plus près du secret des cœurs? Nouvelles lectures historiques des écrits du for privé, Paris, Presses de l'université de Paris-Sorbonne, 2005.

14 Par exemple : Yves Marion, Madeleine Deries (1895-1924), première docteure "ès histoire", Caen, Presses universitaires de Caen, 2017 (chapitres 3 et 4 sur sa scolarité primaire et secondaire, p. 59 et suiv., fondés sur de nombreuses archives familiales).

15 Philippe Marchand, Donnez-moi des nouvelles... Collèges et collégiens à travers les correspondances familiales (1767-1787), Villeneuve d'Ascq, Presses universitaires du Septentrion, 2018. 
livre ici une étude sur les "Souvenirs d'anciens élèves, de la Renaissance à la fin de l'ancien régime scolaire : esquisse pour une analyse historienne". Il montre ainsi, au-delà de la dimension unique de chaque récit de vie, tout ce que peut apporter l'étude croisée de 619 souvenirs, récits d'enfance, et autobiographies. La mise en série des données recueillies, le croisement des parcours individuels recomposés, sur la longue durée, avec la possibilité de dégager des constantes et des évolutions, selon les périodes prises en considération et les thématiques choisies (rôle des précepteurs, processus d'apprentissage de la lecture et de l'écriture, de l'histoire, de la religion, etc.) ouvrent des potentialités multiples d'analyses pour une meilleure connaissance de ce temps de l'élève.

\section{Les enquêtes statistiques du ministère}

Plus ou moins régulièrement depuis le $\mathrm{XIX}^{\mathrm{e}}$ siècle pour le moins, à mesure que l'État affirme ses prérogatives sur l'instruction ${ }^{16}$, le ministère de l'Instruction publique-Éducation nationale, se dote d'outils lui permettant de mieux connaitre l'état des écoles du pays, lançant en particulier de grandes enquêtes statistiques nationales. Il fabrique ainsi ses propres moyens d'observation et d'évaluation des politiques scolaires ${ }^{17}$. L'article proposé ici par Jean-Paul Caille (Direction de l'évaluation, de la prospective et de la performance) et par l'historien Jérôme Krop s'intéresse à une source négligée de l'histoire des élèves : les panels d'élèves des années 1960 aux années 2000. Ces statistiques sont longtemps segmentées entre les trois ordres (primaire, secondaire, supérieur) et n'existent que de manière discontinue. La création du Bureau universitaire de statistique (1933), les travaux lancés par l'INED (Institut national d'études démographiques) après 1946, ceux opérés par le service central de statistiques et de la conjoncture (à partir de 1964) puis par la Direction de l'évaluation et

16 Exemples d'enquêtes sur le secondaire : Ministre de l'Instruction publique (Villemain), Rapport au Roi sur l'instruction secondaire, Paris, Imprimerie royale, 1843, 358 p. (contient une analyse par le ministre, p.3-64, puis des tableaux des principales statistiques sur cet enseignement); Statistique de l'enseignement secondaire en 1865, Paris, Imprimerie impériale, 1868, 481 p.; Statistique de l'enseignement secondaire en 1876, Paris, Imprimerie nationale, 1878, 470 p.; Statistique de l'enseignement secondaire en 1887. Première partie. Enseignement secondaire des garçons, Paris, Imprimerie nationale, 1889, 523 p.; Deuxième partie. Enseignement secondaire des jeunes filles, 259 p.

17 Jean-Noël Luc, La statistique de l'enseignement primaire (19e-20 siècles). Politique et mode d'emploi, Paris, Economica-INRP, 1985; Jean-Pierre Briand, Jean-Michel Chapoulie, Françoise Huguet, Jean-Noël Luc, Antoine Prost, L'enseignement primaire et ses extensions. Annuaire statistique (19e$20^{e}$ siècles). Écoles maternelles, primaires, primaires supérieures et professionnelles, Paris-EconomicaINRP, 1987; Xavier Pons, "Les statisticiens du ministère de l'Éducation nationale : évolutions d'un métier d'État (1957-2007), in Jean-Noël Luc, Philippe Savoie (dir.), L'État et l'éducation en France (XIX ${ }^{\mathrm{e}}$-XX ${ }^{\mathrm{e}}$ siècles), Histoire de l'éducation, ñ $140-141,2014$, p. 115-132. 
de la prospective (DEP) à partir de 1987, devenue la DEPP en 2006, offrent à l'historien des ressources multiples. L'article s'intéresse aux panels, c'est-à-dire aux échantillons d'élèves et de collégiens suivis pendant plusieurs années afin de connaitre leurs parcours scolaires et les facteurs qui influent sur ces parcours, choisis ou subis. Huit enquêtes par panel ont ainsi été lancées depuis 1972, trois sur des panels élèves du primaire et cinq sur des collégiens. Elles permettent de mieux saisir les effets des grandes réformes décidées durant cette période majeure de notre histoire scolaire que sont les années 1970-2000, autour de la réforme Haby du collège unique $(1975)^{18}$, de la création de la politique d'éducation prioritaire par le lancement des zones d'éducation prioritaire (ZEP) par Alain Savary en 1981, puis de ses transformations progressives au cours des années 1990 et 2000, tout en permettant aussi d'étudier les effets de loi Jospin (10 juillet 1989) qui se donne pour objectif d'amener 80 \% d'une classe d'âge au niveau du baccalauréat ${ }^{19}$. On perçoit aussi une évolution progressive dans les objectifs de ces enquêtes par panel. Les premières sont essentiellement centrées sur les parcours des élèves, sur les paliers d'orientation et les choix d'orientation corrélés aux origines sociales et géographiques, la question de la démocratisation étant au cœur des problématiques. À partir des années 1990, en lien aussi avec l'affirmation d'une nouvelle gouvernance et du pilotage par les résultats, les enquêtes cherchent davantage à mesurer les acquis des élèves et leurs compétences. Des pratiques nouvelles de collecte des données sont aussi mises au point qui se tournent vers les familles (leurs représentations et leurs attentes) mais aussi vers les élèves eux-mêmes à partir de 1991.

\section{L'historien face aux sources sur les élèves}

Les sources existent donc bien sur les élèves mais sont marquées par leur éclatement et leur dimension souvent lacunaire ou indirecte. Dès lors, une forte rigueur méthodologique s'impose à l'historien.

18 Jean-Paul Delahaye, Le collège unique pour quoi faire? Les élèves en difficulté au cœur de la question, Paris, Retz, 2006; François Dubet, Pierre Merle, Réformer le collège, Paris, Presses universitaires de France, 2016; Laurent Gutierrez, Patricia Legris (dir.), Le collège unique. Éclairages socio-historiques sur la loi du 11 juillet 1975, Rennes, Presses universitaires de Rennes, 2016.

19 Antoine Prost, L'enseignement s'est-il démocratisé? Les élèves des lycées et collèges de l'agglomération d'Orléans de 1945 à 1990, Paris, Presses universitaires de France, 1992; André D. Robert, L'École en France de 1945 à nos jours, Grenoble, Presses universitaires de Grenoble, 2010; Antoine Prost, Du changement dans l'École, Les réformes de l'éducation de 1936 à nos jours, Paris, Seuil, 2013. 


\section{Les apports d'une mise en corpus des récits de vie}

L'usage d'exemplification ou d'illustration joué par un extrait de récit de vie bien choisi, intégré dans une démonstration plus générale, est fréquent chez les historiens mais pose immédiatement la question de sa représentativité. Pierre Caspard, dans l'article proposé ici, montre au contraire l'intérêt de la constitution d'un corpus et de la mise en série de ces souvenirs d'élèves qui deviennent les sources principales de l'étude. C'est ainsi qu'il présente et analyse plus de 600 souvenirs. Se différenciant des récits littéraires composés par les romanciers, qui mettent souvent en scène les années d'école de leurs personnages (on pense ici à Balzac, Flaubert, Daudet, Zola, etc.) pour mieux en expliquer certains de leurs traits de caractère, de leurs qualités et de leurs défauts, ces souvenirs, mémoires, autobiographies et témoignages mettent en place un régime de vérité qui présente les faits rapportés comme étant vrais. L'étude des origines sociales et professionnelles des auteurs de ces souvenirs montre des origines plus diverses qu'on ne le pense et l'on assiste assez rapidement à un déclin de la présence des milieux nobiliaires (plus prononcé chez les hommes) alors que s'affirme la présence des membres de la bourgeoisie (commerçants, artisans, rentiers, industriels) mais aussi l'existence du "peuple travaillant de ses mains". La gamme des métiers pratiquée est très large, même si Pierre Caspard montre aussi les difficultés de la mise en place d'une typologie (Fautil classer Jules Michelet chez les historiens, les écrivains ou les professeurs?) et les évolutions délicates à suivre de la nomenclature des professions. Les milieux populaires, en particulier ceux des artisans et des ouvriers, sont bien présents, souvent par une élite instruite et militante, alors que la présence paysanne est plus rare. Cette mise en corpus d'une source spécifique obéit, dès lors en partie, aux mêmes logiques scientifiques que celles qui président à la mise en série des parcours individuels au sein d'un groupe humain donné et qui débouchent sur le travail socio-prosopographique, si fécond lui aussi en histoire de l'éducation ${ }^{20}$.

20 Laurent Rollet, Philippe Nabonnand (dir.), Les uns et les autres... Biographies et prosopographies en histoire des sciences, Nancy, Presses universitaires de Lorraine, 2013; Voir par exemple : Christophe Charle, Les professeurs de la Faculté des lettres de Paris. Dictionnaire biographique, t. 1 (1809-1908), Paris, INRP-CNRS, 1985; t. 2 (1909-1939), Paris, INRP-CNRS, 1986; Christophe Charle, Éva Telkes, Les professeurs de la Faculté des sciences de Paris. Dictionnaire biographique (1901-1939), Paris, INRP-CNRS, 1989. 


\section{Des sources à confronter}

Ces récits de vie peuvent être confrontés à d'autres sources. La contribution de Dominique Julia sur les études des princes d'Orléans sous l'autorité de Madame de Genlis (1746-1830) propose un inventaire impressionnant de sources qui permettent de reconstituer le système mis en place par Stéphanie Félicité de Genlis pour éduquer au mieux Louis Philippe d'Orléans, ses deux frères et sa sœur. Nommée "gouverneur" de l'éducation princière en 1782, événement considérable pour une femme et qui n'est pas sans susciter les jalousies, jusqu'à son renvoi en 1791, Madame de Genlis écrit beaucoup et fait beaucoup écrire, l'étude de Dominique Julia proposant un suivi de ces écrits épars qui subissent également une histoire très complexe au gré des événements révolutionnaires et des migrations des familles concernées. Comme le montre Dominique Julia, on est face à "un immense atelier d'écriture", tant Madame de Genlis et son entourage écrivent de journaux, de lettres, de mémoires, de manuscrits. On dispose aussi du journal d'éducation de Charles Gardeur-Lebrun, précepteur des princes après 1785 et sous-gouverneur après l'arrivée de Madame de Genlis. L'article de Dominique Julia propose ici un modèle d'étude de cas permettant de reconstituer la nébuleuse documentaire qui associe sources archivistiques et sources imprimées, au service d'une étude dynamique des processus d'éducation dans un milieu spécifique.

\section{Bien tenir compte de l'intentionnalité des sources utilisées}

Les contributions proposées dans ce numéro invitent également les historiennes et les historiens à bien prendre en considération la nature des documents qu'ils utilisent, comme ils doivent le faire pour n'importe quel sujet. Une première impression de sympathie, parfois teintée de nostalgie pour l'enfance ou la jeunesse que l'on étudie, un sentiment de sincérité issu de traces du passé venant d'un jeune élève, moins soupçonné qu'un adulte de vouloir se mettre en scène et de jouer un rôle réclamé de lui par la société des adultes, peuvent parfois prendre à défaut l'esprit critique historien. Il en va de même du pacte de vérité sur lequel reposent les souvenirs d'enfance et de jeunesse qu'étudie ici Pierre Caspard. Ces autobiographies, ces souvenirs, ces mémoires sont censés décrire ce qui s'est réellement passé à la différence des histoires romancées proposées par la littérature. Mais ces récits de soi, on le sait bien, sont souvent composés au soir de sa vie, lorsque l'auteur est arrivé à un certain âge et occupe une 
certaine position familiale et sociale. Le travail d'écriture est alors une alchimie complexe entre souvenirs réels, recompositions mémorielles, conscientes ou inconscientes, avec parfois d'ailleurs un appui sur des documents conservés. Beaucoup de choses se jouent dans la mise en cohérence des "brouillons de soi $»^{21}$, dans la volonté de donner un sens ${ }^{22}$ ou du sens à sa vie ${ }^{23}$, de justifier certains choix, d'en taire certains autres ${ }^{24}$.

Les souvenirs de scolarité écrits bien après le temps de l'école ne sont donc pas plus "neutres" ou plus marqués par l'innocence que d'autres témoignages. Ceux qui sont écrits à chaud, alors que l'élève est élève, beaucoup plus rares, témoignent eux aussi de formes évidentes de subjectivité, de représentations d'une époque, d'un état scolaire et social précis que l'historien doit expliciter. Les témoignages délivrés par les élèves et recueillis par des adultes, pendant ou juste après des événements importants ${ }^{25}$, ont le même besoin d'examen par la raison critique. Solenn Huitric, dans son étude sur «l'élève au prisme de l'administration", analysant les archives qui concernent les collèges et les lycées entre 1830 et 1880, insiste sur la logique administrative qui préside à leur production, tout chef d'établissement ou inspecteur sachant bien que, lorsqu'il écrit des rapports réguliers sur son établissement ou sur son espace géographique d'affectation, il se donne à voir et donne à voir son établissement ou sa circonscription à ses supérieurs hiérarchiques qui le jugent en même temps. Dès lors, trois thématiques s'affirment constamment dans les remontées documentaires vers le centre de la pyramide administrative. On s'intéresse d'abord à l'évolution des effectifs d'élèves par établissement, par catégories (internes/externes, etc.) et par niveaux car il s'agit d'un élément vital pour connaître la situation financière de l'établissement et sa bonne santé ou son

21 Pierre Lejeune, Le pacte autobiographique, Paris, Seuil, rééd. 1996; Id., Les brouillons de soi, Paris, Seuil, 1998; Id., Autogenèses. Les brouillons de soi-2, Paris, Seuil, 2013.

22 Pierre Bourdieu, "L'illusion biographique", Actes de la Recherche en sciences sociales, 1986, vol. 62-1, p. 69-72.

23 Christine Delory-Momberger, Christophe Niewiadomski (dir.), La mise en récit de soi : place de la recherche biographique dans les sciences humaines et sociales, Villeneuve d'Ascq, Presses universitaires du Septentrion, 2013.

24 François Dosse, Le pari biographique. Écrire une vie, Paris, La Découverte, 2011.

25 Gérard Vincent, Les lycéens. Contribution à l'étude du milieu scolaire, Paris, Armand Colin, 1971; Id., Le peuple lycéen. Enquête sur les élèves de l'enseignement secondaire, Paris, Gallimard, 1974; Didier Leschi, Robi Morder, Quand les lycéens prenaient la parole. Les années 1968, Paris, Syllepse, 2018. 
état de crise ${ }^{26}$. L'élève est bien présent ici mais comme unité de compte. La seconde thématique prioritaire est celle des moyens humains liés à la présence ou à l'absence des enseignants et des maîtres d'études et à leur investissement dans leur travail. La troisième thématique est celle de la vie quotidienne avec un état des lieux sur l'état sanitaire de l'établissement mais aussi sur la discipline et les punitions. L'élève n'est ici présent que lorsqu'il a transgressé les règles écrites et non écrites du collège ou du lycée. Cette prise en compte de l'intentionnalité première des sources utilisées est importante à connaître pour éviter les généralisations abusives.

\section{Retrouver l'élève et son histoire}

Les cinq contributions réunies ici, permettent de dégager trois questions principales qui sont au cœur des recherches actuelles sur l'histoire des élèves.

\section{L'éducation observée au travers du prisme de l'élève : un faisceau d'acteurs}

En plaçant la focale sur les élèves, les textes montrent que les acteurs qui interviennent dans l'éducation-instruction sont multiples sous l'Ancien Régime comme pour la période contemporaine et qu'il faut tenir compte de cette pluralité d'intervenants qui peuvent être complémentaires ou concurrents. Les récits d'élèves étudiés par Pierre Caspard témoignent du grand nombre d'acteurs qui interviennent dans le processus éducatif de la Renaissance au milieu du XIX ${ }^{\mathrm{e}}$ siècle. La famille, les précepteurs, souvent nombreux au cours de l'enfance, les établissements scolaires dans leurs grande diversité, publics ou privés, séminaires, collèges municipaux ou lycées au cours du XIX ${ }^{e}$ siècle, mais aussi les couvents et les pensions particulières pour les filles ${ }^{27}$, jouent des rôles différents selon les périodes et les milieux sociaux. Les souvenirs montrent également des parcours scolaires qui sont plus composites qu'on ne l'a longtemps pensé, avec des usages évolutifs des structures éducatives. La figure

26 Solenn Huitric, Transformer les collèges communaux en lycées. La coproduction d'une action publique (1830-1880), thèse de doctorat, Lyon, université de Lyon-ENS de Lyon, 2016.

27 Martine Sonnet, L'éducation des filles au temps des Lumières, Paris, Cerf, 1987, rééd. 2011; Françoise Mayeur, L'éducation des filles en France au XIX ${ }^{e}$ siècle, Paris, Hachette, 1979; Rebecca Rogers, Les bourgeoises au pensionnat.L'éducation féminine au XIX ${ }^{e}$ siècle, Rennes, Presses universitaires de Rennes, 2007. 
du précepteur ressort nettement des souvenirs d'élèves étudiés ${ }^{28}$, dressant une galerie de portraits très contrastés entre de très bons pédagogues, des escrocs et des personnes peu instruites, dans "un marché préceptoral dépourvu de toutes certifications" comme le note Pierre Caspard. À partir de 1802, l'affirmation de l'État enseignant avec ses cadres, ses règlements, ses méthodes et ses contenus définis, norme progressivement les souvenirs scolaires et les récits de vie étudiés deviennent plus pauvres, décrivant surtout la vie scolaire et les enseignants. La contribution de Jean Le Bihan sur les boursiers communaux des collèges et lycées au XIX ${ }^{e}$ siècle confirme avec force que l'École n'est pas devenue qu'une affaire d'État ${ }^{29}$ et que les communes continuent de jouer un rôle important même dans l'enseignement secondaire souvent perçu comme très lié à l'État. L'examen plus large des réalités éducatives par le prisme du coût des études ${ }^{30}$ (qui finance les structures scolaires, de quelle manière et pour quelles finalités?), avait confirmé cette mobilisation d'acteurs nombreux et concurrents, tout comme les études consacrées à la prise de décision en éducation ${ }^{31}$.

\section{2. Étudier l'élève : un révélateur des articulations entre volontés du pouvoir central et réalités locales}

Retrouver l'élève et la vie qu'il mène dans ses routines et ses ruptures, permet également de réexaminer, au plus près des sources, les relations complexes qui se nouent entre les réalités éducatives locales et les volontés du pouvoir central ${ }^{32}$. L'étude de Jean Le Bihan sur les boursiers communaux au XIX ${ }^{\mathrm{e}}$ siècle permet de saisir les logiques parfois convergentes et parfois divergentes entre l'État et les intérêts locaux. Si les bourses nationales sont à la fois un outil de promotion sociale, souvent destinées aux fils de fonctionnaires et de militaires, l'État

28 Jean-Luc Le Cam (dir.), Éducation privée et pratiques préceptorales du XV au XIX ${ }^{\mathrm{e}}$ siècle, vol. 1, Histoire de l'éducation, $\mathrm{n}^{\circ}$ 143, 2015; vol. 2, Histoire de l'éducation, $\mathrm{n}^{\circ}$ 144, 2015.

29 Jean-Michel Chapoulie, L'École d'État conquiert la France. Deux siècles de politique scolaire, Rennes, Presses universitaires de Rennes, 2010.

30 Jean-François Condette (dir.), Le coût des études : modalités, acteurs et implications sociales (XVI ${ }^{e-}$ XX $X^{e}$ siècle), Rennes, Presses universitaires de Rennes, 2012.

31 Véronique Castagnet-Lars, Caroline Barrera (dir.), Décider en éducation. Entre normes institutionnelles et pratiques des acteurs du XV $V^{e}$ siècle à nos jours, Villeneuve d'Ascq, Presses universitaires du Septentrion, 2019.

32 Jean-François Condette, Les recteurs d'académie en France de 1808 à 1940. T. 3. Le Recteur d'académie, l'École, la Nation (1808-1940). Entre réalités scolaires locales et politiques éducatives nationales, Lyon, INRP, 2009. 
récompensant d'abord ses plus fidèles agents, et un moyen de régulation des flux entre établissements (donner des élèves et de bons élèves à certains établissements) ${ }^{33}$, les boursiers communaux, au travers de l'étude faite par l'auteur de ceux du lycée de Rennes, semblent avoir des profils légèrement différents, avec moins de fils d'agents de l'État et plus d'enfants de cadres moyens et inférieurs de la société (artisans, commerçants, salariés) qui visent à intégrer plus rapidement le monde de l'emploi. Les résultats scolaires sont aussi souvent un peu moins brillants que ceux des boursiers d'État, sur-sélectionnés, alors que le rapport des boursiers communaux au local est plus étroit (être élève de la ville), ainsi que la proximité avec les élus. Marc Suteau dans son étude sur Une ville et ses écoles : Nantes (1830-1940)34 avait déjà bien expliqué ces enjeux contradictoires entre volontés d'État et intérêts locaux. La municipalité soutient ainsi longtemps bien plus l'école primaire supérieure qui fournit des techniciens et des cadres à ses activités économiques portuaires (chantier naval, conserverie, etc.) que le lycée aux études trop généralistes et humanistes, aux débouchés plus lointains.

Les nombreux débats sur la crise de l'enseignement secondaire masculin, à la fin du XIX ${ }^{\mathrm{e}}$ siècle, qui débouchent sur l'enquête parlementaire de $1899^{35}$ et sur la réforme des études de 1902, analysés en particulier par Antoine Prost ${ }^{36}$, parmi plusieurs explications possibles à la stagnation sinon à la baisse des effectifs d'élèves (la crise de l'internat, les tarifs trop élevés, la concurrence des structures privées, etc.), soulignent aussi la préférence de nombreux acteurs locaux pour des études plus pratiques et plus directement liées à l'insertion professionnelle. Ils recommandent alors les études comprenant une maitrise des langues vivantes, réellement parlées, une maîtrise du dessin et des règles de la comptabilité, éléments que l'on trouve d'abord dans les écoles primaires supérieures ${ }^{37}$. Dans

33 Philippe Savoie, La construction de l'enseignement secondaire (1802-1914), Lyon, ENS Éditions, 2013.

34 Marc Suteau, Une ville et ses écoles. Nantes (1830-1940), Rennes, Presses universitaires de Rennes, 1999.

35 Chambre des députés, Enquête sur l'enseignement secondaire, Paris, Imprimerie nationale-Motteroz, 1899, t. 1 et 2 : Procès-verbaux des dépositions, 594 et 719 p.; t. 3 : Statistique et rapports des recteurs et des inspecteurs d'académie, 491 p.; t. 4 : Réponses au questionnaire, 464 p.; t. 5 : Avis des chambres de commerce; délibérations des conseils généraux, 699 p.; t.6 : Rapport général par Monsieur Ribot, 85 p.

36 Antoine Prost, "De l'enquête à la réforme. L'enseignement secondaire des garçons de 1898 à 1902 ", Histoire de l'éducation, $\mathrm{n}^{\circ}$ 119, 2008, p. 29-80.

37 Jean-Pierre Briand, Jean-Michel Chapoulie, Les collèges du peuple. L'enseignement primaire supérieur et le développement de la scolarisation prolongée sous la Troisième République, Paris, INRP-CNRS, 1992. 
l'enquête parlementaire de 1899, les témoignages des chambres de commerce et des conseils généraux ${ }^{38}$ sont très éclairants sur ces priorités divergentes données aux structures scolaires. C'est ainsi que la chambre de commerce d'Abbeville (Somme), par la déposition de son président datée du 10 avril 1899, insiste sur le fait que l'enseignement secondaire classique des collèges et des lycées doit être maintenu en l'état pour former une élite, mais qu'il ne doit pas concentrer l'ensemble des bourses attribuées aux élèves ${ }^{39}$. L'enseignement secondaire moderne n'est "qu'un pâle déguisement de l'enseignement primaire supérieur" et il vaut mieux investir dans ce dernier. "Nous demandons que les concours les plus sérieux puissent seuls donner accès à l'enseignement secondaire et que l'enseignement primaire supérieur reste plus aisément ouvert à ceux que, pour une raison quelconque, l'État tient à protéger. C'est là surtout que se forme cette élite d'employés et de jeunes commerçants capable de rendre par ses études mêmes, de sérieux services au commerce et à l'industrie ${ }^{40}$.

Solenn Huitric, dans l'article proposé ici mais aussi dans sa thèse soutenue en $2016^{41}$, insiste sur le fait que l'enseignement secondaire du XIX siècle demeure une école des notables, mais elle démontre aussi que les profils des établissements secondaires sont beaucoup plus variés qu'on ne l'a longtemps dit, formant un monde composite qui doit s'adapter aux caractéristiques du territoire qui les porte, tout en respectant le cadre normatif imposé par l'Université héritée de Napoléon $1^{\text {er }}$ et incarné par ses nombreux règlements. Les familles ne sont pas passives face aux choix d'orientation et font des calculs, se renseignent sur les établissements existants dans leur zone géographique plus ou moins proche, selon leurs volontés éducatives et l'état de leur fortune. Ce n'est donc pas le centre parisien qui dicte ici sa loi et les choix sont bien locaux, même si l'historien-ne peine à retrouver dans les sources conservées, les déterminants des choix scolaires opérés par les familles entre le collège communal proche, le lycée plus lointain et la pension privée. Les grilles de lecture de l'administration telles qu'elles apparaissent dans les rapports demandés figent les élèves dans un état à un moment donné et ne permettent pas, bien

38 Chambre des députés, Enquête sur l'enseignement secondaire, Paris, Imprimerie nationale-Motteroz, 1899, t. 5 : Avis des chambres de commerce; délibérations des conseils généraux, 699 p.

39 Ibid., Chambre de commerce d'Abbeville, p.2-4.

40 Ibid., p. 4.

41 Solenn Huitric, Transformer les collèges communaux en lycées. La coproduction d'une action publique (1830-1880), thèse de doctorat, Lyon, université de Lyon-ENS de Lyon, 2016. 
souvent, de décrire la réalité de mouvements plus nombreux qu'on ne le pense. Les "chaires ennemies ${ }^{42}$ " n'empêchent pas localement des circulations d'élèves entre institutions qui échappent souvent au regard de l'historien. Dans la ville de Saint-Étienne, étudiée par Solenn Huitric, le collège communal, transformée en lycée en 1840, doit faire face après 1850 à l'Institution Saint-Charles, qui devient ensuite l'institution Saint-Michel (collège jésuite). Il est alors fréquent de trouver dans les rapports du proviseur du lycée mention d'une concurrence très forte entre les deux établissements, la concurrence jésuite devenant un argument récurrent mais un peu facile pour expliquer les baisses passagères d'effectifs. En réalité, une étude conjointe des effectifs des deux établissements montre des évolutions statistiques comparables aux mêmes moments. L'étude du parcours des élèves, au plus près des sources locales conservées, permet, dès lors, d'enrichir fortement l'analyse des relations entre le central et le local.

\section{Les règles de vie, les contenus scolaires et les méthodes pédagogiques}

Même si ces thématiques ne sont pas au cœur de ce numéro d'Histoire de l'éducation, les cinq contributions réunies ayant d'abord pour but de montrer la diversité des sources mobilisables dans l'étude des élèves et les modalités méthodologiques d'analyse de ces sources, il n'en demeure pas moins que l'on perçoit déjà la richesse des études proposées dans l'examen des règles de la vie scolaire, dans l'analyse des contenus scolaires mobilisés et dans les pédagogies usitées. Les récits d'élèves étudiés par Pierre Caspard témoignent ainsi de l'enrichissement progressif des contenus scolaires proposés aux élèves, qu'ils demeurent dans leur famille, sous l'autorité d'un précepteur ou qu'ils rejoignent un établissement scolaire. L'apprentissage de la lecture est souvent très précisément décrit, laissant dans la mémoire des diaristes de forts souvenirs des premières lectures. Cet apprentissage a lieu entre 5 et 7 ans (pour les $2 / 3$ des cas étudiés) et s'opère bien souvent au sein de la famille auprès de la mère, du père ou de la gouvernante. La contribution de Dominique Julia sur les études des princes d'Orléans sous l'autorité de Madame de Genlis permet d'entrevoir des analyses fructueuses sur les études de ces enfants, leurs contenus disciplinaires et les méthodes pédagogiques employées. On devine

42 Sylvain Milbach, Les chaires ennemies. L'Église, l'État et la liberté de l'enseignement secondaire dans la France des notables (1830-1850), Paris, Honoré Champion, 2015. 
aussi l'importance de leur éducation religieuse et la très forte police des mœurs qui est mise en place. Les résumés des activités pédagogiques sont aussi très précis et permettent de suivre le travail, discipline par discipline, et de mesurer les progrès accomplis et les difficultés rencontrées. Les procédés didactiques employés sont aussi perceptibles et Madame de Genlis fait ainsi lire et corriger de nombreuses pièces de théâtre de qualité assez médiocre à ses élèves. On y perçoit aussi les bruits du dehors qui viennent enrichir ou perturber l'étude des princes, comme les événements révolutionnaires à partir de mai 1789. On retrouve ici la richesse des sources qui, aux $\mathrm{XIX}^{\mathrm{e}}$ et $\mathrm{XX}^{\mathrm{e}}$ siècles ensuite, permettent de saisir le travail des élèves à leur échelle, non par les programmes et les manuels scolaires composés par des adultes, mais par les traces conservées de leur mise en activités que sont les copies, les cahiers et les dessins d'élèves ${ }^{43}$.

Alors que l'on ne cesse, de nos jours, de vouloir placer l'élève au "centre du système éducatif", pour en faire le pivot autour duquel doit se réorganiser le système éducatif - l'École étant d'abord et avant tout organisée pour que cet élève puisse réussir - il apparaît important, pour ne pas sombrer dans une forme de présentisme, de mieux connaître et comprendre l'histoire des élèves en tant qu'acteurs à part entière de structures éducatives nombreuses et diversifiées qui ont peu à peu maillé le territoire national, en prenant en compte également les spécificités locales.

Jean-François Condette COMUE-Lille-Nord-de-France (ESPE) -Centre de recherche et d'études Histoire et société -Université d'Artois jeanfrancois.condette@wanadoo.fr

Véronique Castagnet-Lars ESPE-Université de Toulouse Jean Jaurès-FRAMESPA UMR 5136 veronique.castagnet-lars@univ-tlse2.fr

43 Voir en particulier : Pierre Caspard (dir.), "Travaux d'élèves, Pour une histoire des performances scolaires et de leur évaluation (1720-1830)", Histoire de l'éducation, n 46, 1990; Id. (dir.), "Travaux d'élèves, Pour une histoire des performances scolaires et de leur évaluation (XIX ${ }^{e}$-XXe siècles)", Histoire de l'éducation, n ${ }^{\circ}$ 54, 1992; André Chervel, Danièle Manesse, La dictée. Les Français et l'orthographe (1873-1987), Paris, Calmann-Lévy/INRP, 1989; Brigitte Dancel, Enseigner l'histoire à l'école primaire de la III République, Paris, Presses universitaires de France, 1996; Marie-France Bishop, "Racontez vos vacances...". Histoire des écrits de soi à l'école primaire (1882-2002), Grenoble, Presses universitaires de Grenoble, 2010. 